Ashourpouri, A. and Ghosh, A. and Rajakaruna, S. 2018. Droop based Demand Dispatch for Residential Loads in Smart Grid Application. International Journal of Emerging Electric Power Systems. 19 (3): pp. Article number 20170218.

\title{
Droop based Demand Dispatch for Residential Loads in Smart Grid Application
}

Abstract - Aggregated loads play a significant role in maintaining the frequency of power system when the generation is not able to follow frequency deviations. An automatic Demand Dispatch (DD) enables the power system to employ the aggregated loads for balancing demand and supply. In this paper, a Demand Side Frequency Droop (DSFD) has been proposed which provides ancillary service to the grid and maintains the frequency of the power system when the generation system is not capable of following the demand. At the time of a frequency fall/rise, Balancing Authority (BA) can detect aggregated load or group of aggregated loads that have power consumption above or below their standard maximum/minimum consumption levels. Then, the BA issues a droop-based signal to the relevant aggregator. Afterwards, the DSFD will be implemented in the aggregator or the group of aggregators to specify the required power consumption amount for bringing the frequency back to its rated level. Subsequently, this signal will be sent to the Appliance Management Unit (AMU) at each participating house. The AMU sends the signal in the form of deferral or interruptible commands to the appliances depending on the priority, availability and the specification of the appliances. It will be demonstrated that the proposed DSFD control maintains the

\section{Introduction}

frequency of the power system within a specified range.

In recent years, several studies have addressed the issue of Demand Side Management (DSM)

Keywords - Aggregated load, Appliance Management Unit (AMU), Balancing Authority

for balancing power generation with consumption [1] [2] [3] [4]. Also, advancements in two-way (BA), Demand Dispatch (DD), Demand Side Frequency Droop (DSFD).

communications and smart meters pave the way for introducing demand side management to the power system scheduling [1] [5]. Although the significant progress has been made in the area of DSM, residential DSM has been neglected due to the variety of available electrical appliances, their relatively low power consumption, the complexity and the diversity of residential load profiles. However, it is expected that in the near future, high penetration of Electrical Vehicles (EV) will drastically change the residential load profile [6] [7]. Furthermore, smart appliances with built-in communication systems are on their way, which will enhance the role of residential DSM [1] [8]. Moreover, the advancement in 
load modelling and load prediction methods can alleviate the residential load profile complexity [2] [912].

DSM can be applied in a centralised way, decentralised way, or the combination of both [13]. In literature, most of the research works are based on the centralized demand control. In [14], a centralised method is introduced to bring the power consumption below the rated level of distribution transformers. However, a purely centralized method is not appropriate when a large number of various loads are in the mix. Because, the detailed control of different levels of aggregated loads will be missed in the central method. Authors in [15] developed a decentralized Home Energy Management (HEM) algorithm by which the consumers can schedule their loads' consumption patterns. A combination of centralized and decentralized load managements, which forms a multi-level DSM, is proposed in [16]. A DSM is usually applied in the form of load curtailment or load deferral. In [17] an optimised time delay for periodic loads is proposed to prevent peak loading. A load interruption based method is proposed in [18] to shift the demand time and bring the power consumption of a house below the required limit through the HEM system.

Furthermore, DSM can perform load shedding to maintain the frequency of the power system in a relatively more cost effective way. Cost-based incentives such as interruption compensation and/or upfront payment can be offered to customers to encourage them to participate in demand management programs [19] [20] [21]. On the other hand, DSM has this potential to act as spinning reserve for the power grid and participate in stabilising the frequency. The frequency control problem is addressed in an isolated power system equipped with a wind turbine in [22].

In this paper, an improved DSM approach has been proposed. The improvement is achieved by employing a Demand Side Frequency Droop (DSFD) in a hierarchical way to implement Demand Dispatch (DD). In traditional system, the spinning reserve service is the provision of electrical power by a spare generator or a group of spare generators at the time of the generation shortage, with a ramp up time of seconds to 10 minutes [23]. However when a power system has a high penetration level of intermittent renewable energy sources (e.g. wind or solar), the change in power generation can occur very rapidly. In such events, an ancillary service like spinning reserve through usually old inefficient alternators may not be adequate. Also, providing the spinning reserve is very costly for the utilities. To 
alleviate this problem, in this paper a demand dispatch scheme is proposed through which controllable aggregated loads can act as a spinning reserve when there is a generation shortfall.

Usually the loads are quick to respond to external signals and they can be switched on or off almost instantaneously. Therefore, a set of loads can be aggregated and this aggregated load can respond to DD signals fast. Also, due to the enhancement in communication networks, the communication delay of today's communication networks in smart grids have been reduce to milliseconds or less. As a consequence, demand dispatched controllable aggregated loads can provide spinning reserve to a power grid where a rapid change in generation can occur.

In this paper, the frequency deviation is considered as a trip signal for triggering the DD schedule. When the frequency deviations are sensed in the power system, Balancing Authorities (BA) recognises the aggregated loads with the power consumption above or below the permitted level and issues droopbased regulation signals to the aggregators to nominate them for participating in DD program. Then, the feasibility of the issued command will be investigated by the aggregators considering the droop characteristic of the aggregated loads. Afterwards, the droop based demand dispatch commands will be sent to Appliance Management Unit (AMU) in the form of interruptible or deferrable signals until the next command from the BA [24]. These commands are issued based on the time of the day, the priority of the consumers, the features of appliances (for example, the max/min limitation for power draw/consumption), and the overall characteristic of the aggregated loads.

In [24], a DD algorithm has been discussed for a single house. In the current paper, this algorithm has been combined with the demand side frequency droop. Additionally, the algorithm proposed here considers a large number of houses - some of them have EVs and some do not. Therefore the novelty of this paper is to use the frequency droop along with the proposed DD algorithm considering the requirements and capabilities of the residential loads and create a fast spinning reserve for the utilities.

The organisation of this paper is as follows. In Section 2, DSFD will be described along with the AMU algorithm. Afterwards, in Section 3 the proposed method will be investigated through the study case. The paper concludes in Section 4. 


\section{Demand side frequency Droop}

In power systems, the active power affects the power angle and subsequently the frequency of power system. Similarly, reactive power has significant influence on voltage difference [25]. Thus, by controlling active and reactive power in supply side, the frequency and voltage of the power system can be adjusted. However, it is not always practical to increase or decrease the power generated by generators as this procedure might be costly and might affect the stability of the power system.

In this paper, frequency-based droop method has been implemented in demand side to maintain the frequency of the power system. Aggregated residential loads have been considered to be flexible in terms of increasing and decreasing their power consumptions.

It has been assumed that each aggregated load has its own characteristics. These characteristics depend on whether the aggregated load is commercial, industrial, or residential. Moreover, the characteristics of the residential loads depend on the type of the residential suburb in which the aggregated load is located. For instance, the households' welfare and wealth level affect the type of the household appliances and the consumption pattern.

In this paper, it is assumed that the characteristics of the aggregated loads are obtained by carrying out pre-study demand management research or by means of historical data. These characteristics can define the nominal power of the aggregated load and the power variation limit.

Considering the power system shown in Fig.1, the hierarchy of the control system and multilevel aggregation of the loads have been shown. BA plays the role of a central controller while the feeders level aggregators and the lower level aggregators can act as decentral controllers. Also, the communication paths have been demonstrated in Fig.1. Assume that a frequency drop occurs in the power system shown in Fig. 1 and the power generation side is not capable of maintaining the power system frequency. In this case, DD can be applied on the demand side. Through communication and smart metering technology BA has all the information regarding the aggregated load and is capable of detecting the relevant aggregated load for DD program. According to the specification of each aggregated load, the demand-side droop will be implemented. Each aggregated load has its own specific maximum and minimum power consumptions which represent the flexibility of the aggregated load in 
changing the demand. They can be obtained through historical data of the power consumption of the aggregated load and the type of appliances which each residential house is equipped with. To employ demand-side droop, droop coefficient of each aggregated load must be calculated which depend on the specification of the aggregated loads. Fig.2 shows the droop characteristic of an aggregated load.

Fig. 1. Hierarchical control for droop based DD.

Fig.2. The droop characteristic of an aggregated load.

According to Fig.2, the droop equation can be written as [26]:

$\omega_{\max }-\omega_{\min }=m_{L} \cdot\left(P_{L-\max }-P_{L-\min }\right)$

where $m_{L}$ is droop coefficient of the aggregated load, $\omega_{\max }$ and $\omega_{\min }$ are the maximum and minimum angular frequency respectively, $P_{L-\max }$ and $P_{L-\min }$ are the maximum and minimum power consumption of the aggregated load respectively.

Also, according to Fig.2:

$\omega_{\max }-\omega_{\min }=2 \times \Delta \omega_{L-\lim }=4 \pi \times \Delta f_{L-\lim }$

where $\Delta \omega_{L-\text {-lim }}$ is the desired angular frequency limit.

Substituting Equation (2) in Equation (1), we get

$m_{L}=\frac{4 \pi \times \Delta f_{L-\lim }}{\left(P_{L-\max }-P_{L-\min }\right)}$

where $\Delta f_{L \text {-lim }}$ is the desired frequency deviation limit.

In this paper, we assume that the rated frequency of $50 \mathrm{~Hz}$ occurs when the power consumption is in the midpoint between the minimum and maximum power of the aggregated load, i.e.,

$P_{L-\text { rated }}=\frac{\left(P_{L-\max }+P_{L-\min }\right)}{2}$

where $P_{L \text {-rated }}$ is termed as the rated power consumption of the aggregated load.

When the frequency drops, frequency deviation will be calculated through

$\Delta f=f_{g}-f_{\text {rated }}$ 
where $\Delta f$ is the frequency deviation from $f_{\text {rated }}$ and $f_{g}$ is the grid frequency.

Using the frequency droop, $2 \pi . \Delta f=m_{L} . \Delta P_{L}$, the feeder-level aggregator calculates the amount of power required to be cut off in demand side as:

$\Delta P_{L}=\frac{2 \pi \times \Delta f}{m_{L}}$

where $\Delta P_{L}$ is the power consumption which is required to be dropped to bring the frequency back to the rated frequency.

In this paper, a droop-based hierarchical control strategy has been employed to implement an automatic DD. The required power consumption drop will be calculated through Equation (6) in feeder's level aggregator and then will be sent to the lower levels aggregators and eventually to the AMU of the houses which participate in DD. Then, the AMU applies these commands, which are in forms of deferral or interruption requests during frequency drop to the corresponding appliances until the next frequency command from BA.

The calculated $\Delta P_{L}$ will be dispatched among the lower level aggregators according to their participation factors which are defined below

$$
A P F_{k}=\frac{P_{A g g_{-} k}}{P_{T_{-} k}}
$$

where $A P F_{k}$ is Aggregator Participation Factor (APF) of the $k^{\text {th }}$ lower level aggregator, $P_{A g g_{-} k}$ is the power consumption of the $k^{\text {th }}$ lower level aggregator, and $P_{T_{-} k}$ is the rated power of the distribution transformer which is considered as the aggregated node.

It has been assumed that an APF higher than 0.8 participate in DD. According to the average power consumption of the houses and the integrators, aggregators with APF less than 0.8 have less flexibility to participate in DD and the most percentages of the power consumption belongs to the noncontrollable appliances.

Therefore, the required power to be reduced in the $k^{\text {th }}$ lower level aggregator can be calculated as 
$\Delta P_{\mathrm{Agg}_{-} k}=\frac{P_{\mathrm{Agg}_{-} k}}{\sum_{k=1}^{n} P_{{\mathrm{Ag} g_{-} k}}} \times \Delta P_{L}$

where $\Delta P_{\text {Agg_k }_{k}}$ is the required power consumption curtailment for the $k^{\text {th }}$ lower level aggregator and $n$ is the number of the lower level aggregators connected to the feeder level aggregators to participate in DD. So, $k$ can change from 1 to $n(k=1,2, \ldots, n)$.

Afterwards, $\Delta P_{A g g_{-} k}$ will be shared among the AMUs according to the participation factor of each house:

$H P F_{k_{-} l}=\frac{P_{H_{-} k_{-} l}}{P_{H_{-} k_{-} l}}$

where $H P F_{k_{-}}, P_{H_{-} k_{-} l}$, and $P_{H M_{-} k_{-}}$are the Home Participation Factor, the power consumption, and the maximum power consumption threshold of the $l^{\text {th }}$ home in the $k^{\text {th }}$ lower level aggregator respectively. $P_{H M_{-} k_{-} l}$ is defined according to the number of the household members, the size of the property and the type of appliances in the household. Similar to the aggregators, the households with HPF higher than 0.8 participate in $\mathrm{DD}$.

Therefore, the required power to be curtailed from the $l^{\text {th }}$ home in the $k^{\text {th }}$ lower level aggregator is

$$
\Delta P_{H_{-} k_{-} l}=\frac{P_{H_{-} k_{-} l}}{\sum_{l=1}^{m} P_{H_{-} k_{-} l}} \times \Delta P_{A g g_{-} k}
$$

where $\Delta P_{H_{-} k_{-} l}$ is the required power consumption curtailment for the $l^{\text {th }}$ house in the $k^{\text {th }}$ lower level aggregator and $m$ is the number of the houses in the $k^{\text {th }}$ lower level aggregator which participates in DD. So, $l$ can change from 1 to $m(l=1,2, \ldots, m)$.

After calculating the required power deduction, it will be issued to the AMU. AMU postpones or switches off the corresponding loads according to the priority and the availability of the appliances. It has been assumed that the power consumption of an entire house and its appliances are measured and monitored every $30 \mathrm{sec}$. Therefore, the DD schedule can be implemented at the appliance level with the help of the AMU that is installed in each house. It should be noted that, the smart house is equipped 
with communication interface and different sensors and measurement devices which enable the AMU system to gather data about room temperature, water temperature, power consumption, status of appliances, load priority, costumer's preference, State of Charge (SoC) of EV batteries, etc. An AMU system has a significant role in performing an automated DD within residential properties and ideally should have the least intrusive effects on consumers. The AMU system for a smart house with controllable appliances is shown in Fig. 3 [24].

Fig. 3. The AMU system for a smart house with controllable appliances.

The signals issued from the AMU to the appliances are in the form of a deferral or interruption commands when the frequency of the system drops. The AMU releases these commands according to the signals received from the lower level aggregator, the priority of the consumers, and the features of appliances.

The proposed AMU algorithm has been shown in Fig. 4. The purpose of this algorithm is to reduce the demand during the peak times[24].

Fig. 4. The Proposed AMU load reduction algorithm.

As illustrated in Fig4., when the frequency signal has been issued to the AMU, it gathers information such as, appliances power consumption $\left(P_{a p p, j}\right)$, overall power consumption of the home $\left(P_{T L}\right)$, status of appliances $\left(S_{a p p, j}\right)$, room temperature $\left(T_{r}\right)$, hot water temperature $\left(T_{H W}\right)$, demand limit $\left(P_{D L}\right)$, duration of demand limit $\left(t_{D L}\right)$, priority and satisfaction of consumers [24].

If the total load power consumption of the house is more than the demand power limit, then the excess power $\left(P_{e x c}\right)$ will be calculated according to

$$
\text { If } P_{T L}>P_{D L} \text { then } P_{e x c}=P_{T L}-P_{D L}
$$

Afterwards, a load or a group of loads will be selected according to the priority and satisfaction levels, as well as considering constraints (12)-(21):

Controllable Appliances:

$$
\sum_{j=1}^{Q} P_{a p p, j} \geq P_{e x c}
$$


where $j$ is the number of selected controllable loads which are required to be delayed or interrupted and $Q$ is the number of controllable appliances. It should be noted that it has been assumed that the maximum value for $Q$ is 8 .

Clothes Dryer (DRY):

$t_{\text {delay,dry }}<23: 00-t_{c y c l e, d r y}-t_{i}$

where $t_{\text {delay,dry }}, t_{c y c l e, d r y}$, and $t_{i}$ are DRY delay time duration (hr), DRY cycle time (hr), and current time slot $i(\mathrm{hr})$ respectively. In this paper, $t_{c y c l e, d r y}$ is chosen as 1 hour. The DRY delay time can be set by the consumer.

EV:

$S o C_{\min } \leq S o C \leq S o C_{\max }$

where $S o C_{\min }$ and $S o C_{\max }$ are minimum and maximum SoC of the battery of EV.

$t_{o f f, e v}<30 \mathrm{~min}$

where $t_{o f f, e v}$ is off time duration of $\mathrm{EV}(\mathrm{min})$.

$N_{S_{-} \text {sofar }, e v} \leq 3$

where $N_{s_{-} \text {sofar,ev }}$ is the number of signals which EV has received to be switched off so far.

In this paper, 3 times has been selected. But this parameter can be changed based on different factors such as consumers' needs and battery requirements.

Pool Pump (PP):

$t_{\text {on_sofar }, p p} \geq 6: 00$

where $t_{o n_{-} \text {sofar,pp }}$ is the accumulated time duration which the PP has been on during the current day.

$t_{\text {delay,pp }} \leq 24: 00-\left(6: 00-t_{\text {on_sofar }, p p_{1}}\right)$

where $t_{\text {delay,pp }}$ is PP delay time duration (hr). This consideration simply means the PP must work at least $6 \mathrm{hrs}$ per day.

Clothes Washer $(\mathrm{CW})$ : 
$t_{\text {delay, }, \mathrm{w}}+t_{\text {cycle }, \mathrm{cw}}<t_{\text {start,dry }}$

where $t_{\text {delay,cw }}$ and $t_{\text {cycle,cw }}$ are $\mathrm{CW}$ delay time duration (hr) and $\mathrm{CW}$ cycle time (hr) respectively. In this paper, $t_{c y c l e, c w}$ is assume to be 40 min. $t_{\text {start }, d r y}$ is starting time of DRY.

$t_{\text {delay,cw }}<23: 00-t_{\text {cycle,cw }}-t_{i}$

where $t_{\text {delay,cw }}$ and $t_{c y c l e, c w}$ are delay time duration (hr) and cycle time (hr) of CW respectively.

Dish Washer (DW):

$t_{\text {delay }, d w}<23: 00-t_{c y c l e, d w}-t_{i}$

where $t_{c y c l e, d w}$ is $2: 00 \mathrm{hr}$.

These constraints shall be considered according to the consumers' satisfactions. If the selected controllable load is deferrable, then the starting time of the appliance will be delayed according to the householders' satisfaction. If the load is interruptible then it will be switched off. Then, the information will be updated at the beginning of the next time slot. Until the next new frequency signal the DD scheme will continue.

\section{Case Study}

As mentioned before, the purpose of this paper is to maintain the frequency of the power system within the acceptable range with the help of the proposed DSFD method and the DD algorithm when any spinning reserve is not available or capable of responding fast enough to the generation rise/drop. Hence, in case of frequency drop, BA detects the feeder-level aggregator with high power consumption and calculates the required power reduction. Then, the feeder-level aggregator calculates the power reduction required for each house. Afterward, the AMU connected to each house interrupts or delays the controllable appliances though DD schedule

To investigate the validity of the proposed DSFD system, the power system shown in Fig. 1 has been considered. Assume that a frequency drop happens at 17:30 hrs due to the peak demand in the afternoon, where the generation side is not capable of responding to this frequency drop. Thus, demand shedding is required to bring the frequency back to its acceptable range $\left[f_{\text {rated }}-\Delta f_{L \text {-lim }}, f_{\text {rated }}+\Delta f_{L-\text { lim }}\right]$. 
The required frequency range and the specifications of the feeder-level aggregated load which has been considered in this study case are shown in Table 1. Different types of houses have been considered in this study. The specifications of two typical houses with/without EV and their satisfaction have been listed in Table 2 and Table 3 respectively. The information in Table 2 and Table 3 can be collected from consumers in time of signing the DD participation contract.

Table 1: Specifications of the feeder's level aggregated load

\begin{tabular}{ll}
\hline Parameter & Value \\
\hline$f_{\text {rated }}$ & $50 \mathrm{~Hz}$ \\
$\Delta f_{L-\text { lim }}$ & $\pm 0.05 \mathrm{~Hz}$ \\
$P_{L-\text { max }}$ & $\mathrm{KW} \mathrm{630}$ \\
$P_{L-\min }$ & $\mathrm{KW} 190$ \\
\hline
\end{tabular}

Table 2: Appliances specifications

\begin{tabular}{|c|c|c|c|c|}
\hline \multicolumn{2}{|c|}{ Appliance } & \multirow{2}{*}{$\begin{array}{l}\text { Type of the Appliance: } \\
\text { 1) Non-controllable } \\
\text { 2)Controllable: a) deferrable } \\
\text { b) interruptible }\end{array}$} & \multicolumn{2}{|c|}{ Rated power (W) } \\
\hline & & & House 1 & House 2 \\
\hline \multirow{2}{*}{$\begin{array}{l}\text { DRY } \\
\text { (Dryer) }\end{array}$} & Rotating Tumble (RT) & non-controllable & 300 & 300 \\
\hline & Heating Coil (HC) & $\begin{array}{l}\text { controllable/interruptible \& } \\
\text { deferrable }\end{array}$ & 3500 & 3000 \\
\hline \multirow{2}{*}{$\begin{array}{l}\text { FR } \\
\text { (Fridge) }\end{array}$} & Defrost Cycle & controllable/ deferrable & 400 & 400 \\
\hline & Fridge Compressor & non-controllable & 150 & 150 \\
\hline \multicolumn{2}{|c|}{ WH (Water Heater) } & controllable/interruptible & 4500 & 4000 \\
\hline \multicolumn{2}{|c|}{ AC (Air Conditioner) } & controllable/interruptible & 1500 & 1400 \\
\hline \multicolumn{2}{|c|}{ EV (Electrical Vehicle) } & $\begin{array}{l}\text { controllable/interruptible \& } \\
\text { deferrable }\end{array}$ & 3300 & N/A \\
\hline \multicolumn{2}{|c|}{ PP (Pool Pump) } & controllable/ deferrable & 1500 & 1400 \\
\hline \multicolumn{2}{|c|}{ CW(Clothes Washer) } & controllable/ deferrable & 600 & 550 \\
\hline \multicolumn{2}{|c|}{ DW(Dishwashers) } & controllable/ deferrable & 1500 & 1400 \\
\hline \multicolumn{2}{|c|}{ KT (Kettle) } & non-controllable & 1500 & 1200 \\
\hline \multicolumn{2}{|c|}{ HD (Hair dryer) } & non-controllable & 1500 & 1300 \\
\hline \multicolumn{2}{|c|}{ LI (Lighting) } & non-controllable & Vary $(50-300)$ & Vary $(40-240)$ \\
\hline \multicolumn{2}{|c|}{ OV (Oven) } & non-controllable & 2000 & 1500 \\
\hline
\end{tabular}




\begin{tabular}{|l|l|l|}
\hline MW (Microwave) & non-controllable & 1100 \\
\hline CM (Coffee Machine) & non-controllable & 1000 \\
\hline TO (Toaster) & non-controllable & 1000 \\
\hline TV & non-controllable & 250 \\
\hline MS (Miscellaneous Appliances such as iron, computers, & non-controllable & Vary (1500- \\
chargers...) & & $3000)$ \\
\hline
\end{tabular}

Table 3: Customer's satisfaction and Priority

\begin{tabular}{|c|c|c|c|c|}
\hline \multirow[t]{2}{*}{ Appliance } & \multicolumn{2}{|c|}{ Priority } & \multicolumn{2}{|l|}{ Preference } \\
\hline & $\begin{array}{l}\text { House } \\
1\end{array}$ & $\begin{array}{l}\text { House } \\
2\end{array}$ & House 1 & House 2 \\
\hline DRY & 4 & 4 & $\begin{array}{l}\text { Finish the tasks before } 23: 00 \text {. } \\
\text { Max } 30 \text { min off time in time of } \\
\text { interruption. }\end{array}$ & $\begin{array}{l}\text { Finish the tasks before } 22: 30 . \\
\text { Max } 30 \text { min off time in time of interruption. }\end{array}$ \\
\hline FR & 7 & 6 & $\begin{array}{l}\text { Defrost cycle can be delayed for up to } \\
3 \mathrm{hrs} \text {. }\end{array}$ & Defrost cycle is not allowed to be delayed. \\
\hline WH & 1 & 1 & $\begin{array}{l}\text { Water temperature must be within } 51- \\
54^{\circ} \mathrm{C} \text {. }\end{array}$ & Water temperature must be within $51-55^{\circ} \mathrm{C}$. \\
\hline $\mathbf{A C}$ & 2 & 2 & $\begin{array}{l}\text { Room temperature must be within } 20 \text { - } \\
22^{\circ} \mathrm{C} \text {. During } \mathrm{DD}, 3^{\circ} \mathrm{C} \text { increase is } \\
\text { acceptable. }\end{array}$ & $\begin{array}{l}\text { Room temperature must be within } 22-24^{\circ} \mathrm{C} . \text { During } \mathrm{DD}, 2^{\circ} \mathrm{C} \\
\text { increase is acceptable. }\end{array}$ \\
\hline $\mathbf{E V}$ & 5 & N/A & $\begin{array}{l}S o C_{\min }=\% 50, \quad S_{o} C_{\max }=\% 100 \\
\text { Fully charged by } 8 \mathrm{am} . \text { Max } 3 \text { times } \\
\text { being switched off during } \\
\text { interruption. }\end{array}$ & N/A \\
\hline $\mathbf{P P}$ & 6 & 5 & Run at least 6 hours in 24 hours. & Run at least 6 hours in 24 hours. \\
\hline $\mathbf{C W}$ & 3 & 4 & $\begin{array}{l}\text { Must finish the task before starting } \\
\text { time of DRY. Finish the task before } \\
\text { 23:00. }\end{array}$ & $\begin{array}{l}\text { Must finish the task before starting time of DRY. Finish the } \\
\text { task before } 22: 30 \text {. }\end{array}$ \\
\hline DW & 3 & 3 & Finish the task before 23:00. & Finish the task before $22: 30$. \\
\hline
\end{tabular}

After sensing the frequency drop in the system, BA detects the feeder level aggregator with the power consumption beyond the stipulated limit. In this study, we assume $\mathrm{SM}_{\mathrm{F}}$ is the detected feeder. It has been assumed that 100 different houses are connected to this feeder. The objective of this study is 
to bring the frequency of the system back to the acceptable range with the help of the proposed DSFD and AMU algorithm.

After detecting the problematic feeder, DSFD will be implemented in the corresponding aggregator and the amount of required power curtailment will be calculated using Equation (6). Then, the lower level aggregators which must participate in DD will be selected by the feeder level aggregator and the amount of power which is required to be shed will be calculated and issued to them. Then the relevant AMU systems will apply the required changes to the consumption of the relevant loads.

Figs. 5 and 6 show the results of the DSFD on the feeder level aggregator power consumption profile and the frequency with/without DSFD. In Fig. 5, the power consumption of the feeder level aggregated load rises above the maximum power consumption limit shown in Table 1, which leads to frequency drop shown in Fig. 6. As shown in Fig. 6, the proposed DSFD method is capable of maintaining the frequency within the range of $50 \pm 0.05 \mathrm{~Hz}$, i.e., between $49.95 \mathrm{~Hz}$ and $50.05 \mathrm{~Hz}$ by reducing the power consumption in the downstream aggregators shown in Fig 5.

Fig 5. The feeder level aggregated load power consumption profile without and with DSFD.

Fig 6. The frequency of the power system without and with DSFD.

Bringing the frequency of the system within the acceptable range is not achievable without the AMU algorithm. After the required power consumption drop has been assigned to each participated house, the AMU algorithm is responsible for reducing the house power consumption. Two different houses as described below have been selected to reflect the effect of the DSFD on the households. House 1 has been considered to be a typical house with EV where the householders are not at home between 8:00 hrs and 17:00 hrs. The EV is plugged in as soon as the householders arrive home. House 2 does not have any EV and the householders are at home during the day.

After the AMUs in the selected houses receive the relevant signals, they postpone or interrupt the operation of the appliances according to the AMU algorithm. Fig.7 and Fig.8 illustrate the load profile of the two houses with/without DSFD. As shown in these two figures, the power limit for house 1 and 2 has been changed at 19:30 hrs because the tension on frequency of the system has been alleviated. At 20:30 hrs, the DSFD signals have been removed. As shown in Figs. 7 and 8, the proposed DSFD method 
with the help of the AMU algorithm is capable of maintaining the power consumption of the houses below the required power limit.

Fig. 7. House 1 power consumption without and with DSFD.

Fig. 8. House 2 power consumption without and with DSFD.

Fig. 9 shows the normal operation of the controllable appliances in house 1 before participating in the DD program. Fig. 10 shows the detailed operation of the same controllable appliances in house 1 after participating in DD. WH load profile has not been illustrated as it has not been selected by AMU to participate in DD due to its high priority. As shown in Fig. 10, the power consumptions of controllable appliances were altered according to AMU algorithm and DSFD. For example, the operation of CW is delayed by 1.5 hours according to equations (17) and (18). The other example of this alteration is AC's temperature and power consumption profile as shown in Fig. 10. During DD event, AC is allowed to increase the room temperature by $3^{\circ} \mathrm{C}$ according to costumer's satisfaction given in Table 3 . The operations of other controllable appliances such as DRY, PP, DW, FR defrost cycle, and EV which participate in DD are also shown in Fig. 10.

Fig. 9. DRY, FR, DW, CW, PP, AC, and EV operation in house 1 without DSFD.

Fig. 10. DRY, FR, DW, CW, PP, AC, and EV operation in house 1 with DSFD.

The operation of the controllable appliances in house 2, which have participated in the DD event are shown in Fig. 11. As mentioned before, there is no EV in house 2. The detailed operation of AC, DRY, DW, and PP before receiving DD signals have been shown in Fig. 11. WH and FR and CW load profiles have not been shown as they did not participate in DD due to the priority of the householders. The altered power consumption of AC, DRY, DW, and PP after receiving DD signals have been shown in Fig. 12. For example, AC's temperature is allowed to rise up to $2^{\circ} \mathrm{C}$ according to the satisfaction level of the householders of house 2 as mentioned in Table 3.

Fig. 11. DRY, DW, PP, and AC operation in house 2 without DSFD.

Fig. 12. DRY, DW, PP, and AC operation in house 2 with DSFD.

Fig. 5-12 have illustrated the cooperative operation of the DSFD and AMU system. DSFD releases the frequency signal to the relevant aggregators after sensing the frequency drop in the power system. Afterwards, the required power consumption curtailments were dispatched to the participating AMUs in each house via lower level aggregator to bring the frequency back to the acceptable limits. 
The AMUs reduce the required power by postponing or curtailing the power consumptions of the appliances according to their characteristic and householders satisfactions.

\section{Conclusion}

The proposed DSFD offers significant improvements on maintaining the frequency of the power system. This paper demonstrated how this method can maintain the frequency of the system within the acceptable range. It has been demonstrated that BA is capable of detecting the relevant aggregators to participate in DD program. Afterwards, by implementing DSFD in aggregators' level, the required power consumption level, which the aggregated loads must consume to bring the frequency back within the rated range, will be calculated. This limited amount of power consumption will be issued to the lower level aggregators and then finally to the AMU system in each house. Afterwards, the AMU algorithm alters the power consumption of the house accordingly by sending the commands in form of deferral or interruptible signals to various appliances. The case study results confirm the suitability of the proposed approach.

\section{References}

[1] A. Brooks, E. Lu, D. Reicher, C. Spirakis, and B. Weihl, "Demand Dispatch," Power and Energy Magazine, IEEE, vol. 8, pp. 2029, 2010.

[2] Y. Zhang, R. Wang, T. Zhang, Y. Liu, and B. Guo, "Model predictive control-based operation management for a residential microgrid with considering forecast uncertainties and demand response strategies," IET Generation, Transmission \& Distribution, vol. 10 , pp. $2367-2378,2016$

[3] S. Barker, S. Kalra, D. Irwin, and P. Shenoy, "Empirical Characterization, Modeling, and Analysis of Smart Meter Data," Selected Areas in Communications, IEEE Journal on, vol. 32, pp. 1312-1327, 2014.

[4] V. S. K. M. Balijepalli, V. Pradhan, S. A. Khaparde, and R. M. Shereef, "Review of demand response under smart grid paradigm," in Innovative Smart Grid Technologies - India (ISGT India), 2011 IEEE PES, 2011, pp. 236-243.

[5] Z. Ziming, S. Lambotharan, C. Woon Hau, and F. Zhong, "Overview of demand management in smart grid and enabling wireless communication technologies," Wireless Communications, IEEE, vol. 19, pp. 48-56, 2012.

[6] A. Ashourpouri, M. Dargahi, and S. A. Nabavi Niaki, "Residential voltage dip and swell mitigation using Plug-in Hybrid Electric Vehicle in smart grid," in Power Engineering Conference (AUPEC), 2013 Australasian Universities, 2013, pp. 1-5.

[7] S. Shengnan, M. Pipattanasomporn, and S. Rahman, "Demand Response as a Load Shaping Tool in an Intelligent Grid With Electric Vehicles," Smart Grid, IEEE Transactions on, vol. 2, pp. 624-631, 2011.

[8] S. A. Pourmousavi and M. H. Nehrir, "Real-Time Central Demand Response for Primary Frequency Regulation in Microgrids," IEEE Transactions on Smart Grid, vol. 3, pp. 1988-1996, 2012.

[9] R. Yao and K. Steemers, "A method of formulating energy load profile for domestic buildings in the UK," Energy and Buildings, vol. 37, pp. 663-671, 6// 2005.

[10] J. Widén, M. Lundh, I. Vassileva, E. Dahlquist, K. Ellegård, and E. Wäckelgård, "Constructing load profiles for household electricity and hot water from time-use data-Modelling approach and validation," Energy and Buildings, vol. 41, pp. 753-768, 7// 2009.

[11] I. Richardson, M. Thomson, D. Infield, and C. Clifford, "Domestic electricity use: A high-resolution energy demand model," Energy and Buildings, vol. 42, pp. 1878-1887, 10// 2010.

[12] M. A. Zehir and M. Bagriyanik, "Demand Side Management by controlling refrigerators and its effects on consumers," Energy Conversion and Management, vol. 64, pp. 238-244, 12// 2012.

[13] G. Benysek, J. Bojarski, M. Jarnut, and R. Smolenski, "Decentralized Active Demand Response (DADR) system for improvement of frequency stability in distribution network," Electric Power Systems Research, vol. 134, pp. 80-87, 2016/05/01/ 2016.

[14] F. Shahnia, M. T. Wishart, A. Ghosh, G. Ledwich, and F. Zare, "Smart demand side management of low-voltage distribution networks using multi-objective decision making," Generation, Transmission \& Distribution, IET, vol. 6, pp. 968-1000, 2012. 
[15] C. Tsung-Hui, M. Alizadeh, and A. Scaglione, "Real-Time Power Balancing Via Decentralized Coordinated Home Energy Scheduling," Smart Grid, IEEE Transactions on, vol. 4, pp. 1490-1504, 2013.

[16] J. Hu, J. Cao, and T. Yong, "Multi-level dispatch control architecture for power systems with demand-side resources," IET Generation, Transmission \& Distribution, vol. 9, pp. 2799-2810, 2015.

[17] G. Koutitas and L. Tassiulas, "A delay based optimization scheme for peak load reduction in the smart grid," in Future Energy Systems: Where Energy, Computing and Communication Meet (e-Energy), 2012 Third International Conference on, 2012, pp. 14.

[18] M. Pipattanasomporn, M. Kuzlu, and S. Rahman, "An Algorithm for Intelligent Home Energy Management and Demand Response Analysis," Smart Grid, IEEE Transactions on, vol. 3, pp. 2166-2173, 2012.

[19] I. U. Nutkani, L. Poh Chiang, and F. Blaabjerg, "Cost-based droop scheme with lower generation costs for microgrids," Power Electronics, IET, vol. 7, pp. 1171-1180, 2014.

[20] M. Fahrioglu and F. L. Alvarado, "Designing incentive compatible contracts for effective demand management," Power Systems, IEEE Transactions on, vol. 15, pp. 1255-1260, 2000.

[21] C. Vivekananthan, Y. Mishra, and K. Rajashekara, "Energy efficient home with price sensitive stochastically programmable TCAs," in Industrial Electronics Society, IECON 2014 - 40th Annual Conference of the IEEE, 2014, pp. 5357-5362.

[22] A. Molina-Garc, x00Ed, I. Mu, x00F, B. oz, A. D. Hansen, et al., "Demand-Side Contribution to Primary Frequency Control With Wind Farm Auxiliary Control," IEEE Transactions on Power Systems, vol. 29, pp. 2391-2399, 2014.

[23] G. Joos, B. T. Ooi, D. McGillis, F. D. Galiana, and R. Marceau, "The potential of distributed generation to provide ancillary services," in 2000 Power Engineering Society Summer Meeting (Cat. No.00CH37134), 2000, pp. 1762-1767 vol. 3.

[24] A. Ashourpouri, A. Ghosh, and S. Rajakaruna, "Demand Dispatch through Appliance Management Unit in a Semi-Smart Home," presented at the Industrial Electronics Society, IECON 2016 - 42st Annual Conference of the IEEE, Florence, Italy, 2016.

[25] P. Kundur, N. J. Balu, and M. G. Lauby, Power system stability and control vol. 7: McGraw-hill New York, 1994.

[26] A. Ashourpouri, A. Ghosh, and F. Shahnia, "Droop-Based Demand Dispatch Schedule In Smart Grid," in Power Engineering Conference (AUPEC), 2015Australasian Universities, 2015, pp. 1-6. 